\title{
REFLEXIONES EN TORNO A LAS COMPETENCIAS PROFESIONALES SOLICITADAS A LOS MAESTROS DE EDUCACIÓN FÍSICA CON LA IMPLANTACIÓN DE LA LOGSE
}

\section{Autor:}

\section{Introducción}

En los planes de estudios para obtener el título de Maestro Especialista en Educación Física siempre ha habido unos créditos que llegaban impuestos a las Escuelas Universitarias de Formación del Profesorado y otros cuya implantación se debatía en la Junta de Centro de dichas Escuelas. Cuando se empezaron a cons truir los estudios de Maestro de Educación Física, Plan del 71 , se señalaban las asignaturas de especialidad a partir de las comunes, aquellas que se impartían en todas las especialidades que contemplaba dicho Plan, eran aproximadamente el $50 \%$ de la carga total. Siguiendo este criterio elaboramos el primer Plan en la Escuela de Formación del Profesorado de EGB en Palencia, que junto con la de León fueron, como es sabido, las primeras en implantar dichos estudios.

En el plan actual, hay asignaturas troncales que todas las Escuelas y Facultades de Educación del país que imparten el título de Maestros/as Especialistas de Educación Física deben respetar. También en este Plan la Junta de Centro de las diferentes Escuelas y Facultades de Educación han tenido que proponer asignaturas obligatorias, optativas y de libre elección para completar y enriquecer la formación prevista en las troncales y obtener Maestros de Educación Física competentes.

En la Escuela Universitaria de Educación de Palencia desde el primer momento, se consideró que debía haber una asignatura dedicada específicamente a formar en y a través de, las tareas que se reclaman a los maestros en el tratamiento educativo del ámbito corporal. Durante un tiempo pudimos hacerlo para las etapas de Infantil y Primaria y en estos momentos, por causa de la reducción continuada de créditos, sólo hay una asignatura obligatoria dedicada expresamente a esta cuestión se denomina: "Educación Física en Educación Primaria". Desde el curso 1989-1990, que se implantan los estudios de Maestros/as Especialistas de Educación Física en la Escuela de Palencia se ha venido impartiendo la asignatura mencionada, al principio con nueve créditos, en estos momentos con seis.

A partir de la implantación de la LOGSE (Ministerio de Educación y Ciencia, 1990) las tareas de los maestros quedaron definidas a nivel de Boletín Oficial: $A$ los equipos docentes y profesores les queda la tarea de elaboración de Proyectos Curriculares y programaciones B.O.E. del 9.09.1991. ANEXO

Para responder a estas exigencias, la formación del profesorado en esta asignatura se centró específicamente en la integración del ámbito corpora en el Proyecto Educativo de Centro, la elaboración de una propuesta curricular que ayude a señalar la Programación General Anual y en el diseño, desarrollo y análisis de unidades didácticas. (Para ampliar datos, véase el Programa de la Asignatura)

La asignatura mencionada se ha impartido siempre en segundo curso, en estrecha relación con las Prácticas I, las específicas, es decir, en las que los estudiantes actúan como Maestros/as Especialistas de Educación Física. Por otra parte, el profesor que la desarrolla participa activamente en el Grupo de Trabajo: "Tratamiento Pedagógico de lo Corporal", que se reúne en la Escuela Universitaria de Educación todos los miércoles y en el que se dan cita un buen grupo de Maestros/as Especialistas de Educación Física y Generalistas cuyo tema de estudio insiste en las actividades previstas en la asignatura mencionada.

Progresivamente, las asignaturas citadas y las actividades que se realizan en el Grupo de Trabajo han ido, adecuándose a las exigencias de la Investigación-Acción (Elliott 1990) estableciendo diferentes ciclos entre su programación, desarrollo y análisis. Uno de estos 
ciclos, el que se corresponde con el curso escolar, posibilita cada año la reformulación a comienzo de curso de los proyectos a desarrollar.

En los informes de final de curso sobre las tareas desarrolladas en el Grupo de Trabajo y en las asignaturas de "Educación Física en Educación Primaria" y "Prácticas I", fuimos detectando la necesidad de identificar cual son las competencias que se reclaman de los maestros en el desarrollo de las tareas oficialmente asignadas.

Como en tantas otras ocasiones, fue el análisis de la práctica educativa el que nos fue proporcionando unos apuntes, sobre los que debatimos una y otra vez hasta elaborar un documento que, en el curso 2000-2001, quedó redactado tal como lo exponemos a continuación:

\section{Sobre las competencias pro- fesionales solicitadas a los maestros de educación física con la implantación de la LOGSE}

COMPETENCIAS RELACIONADAS CON LA ELABORACIÓN, DESARROLLO Y EVALUACIÓN DEL PROYECTO EDUCATIVO DEL CENTRO

Establecer relaciones de colaboración con los maestros tutores que ayuden al conocimiento del alumnado y al desarrollo del potencial educativo del ámbito corporal.

Identificar justificar y proponer contenidos relevantes del ámbito corporal cuyo desarrollo depende de los maestros tutores, del conjunto del profesorado, del plan de actividades extraescolares del centro.

Adecuar las tareas relativas al ámbito corporal a los diferentes lugares de acción educativa que contempla el centro y sus alrededores.

Colaborar con los proyectos educativos que desde el ámbito corporal se desarrolla en el segundo ciclo de Educación Infantil y primero de la Enseñanza Secundaria Obligatoric.

COMPETENCIAS RELATIVAS A LA ELABORACIÓN, DESARROLLO Y EVALUACIÓN DE LA PROPUESTA CURRICULAR

Presentar y justificar una Propuesta Curricular de Educación Física en Educación Primaria, equilibrada, pertinente y comprensiva.

Ajustar los rasgos básicos de la Propuesta Curricular en los diferentes ciclos que forman la Educación Primaria.

Abrir la Propuesta Curricular a la participación del resto del profesorado y de otros agentes educativos.

Establecer instrumentos que faciliten la observación y reconducción de la Propuesta Curricular.
COMPETENCIAS RELACIONADAS CON LA PROGRAMACIÓN DESARROLLO Y ANÁLISIS DE LOS PROCESOS DE ENSEÑANZA-APRENDIZAJE

\section{PROGRAMACIÓN}

Justificar el tema elegido como proceso de enseñanza y aprendizaje, decir por qué merece la pena.

Enunciar claramente los contenidos a aprender y las capacidades a desarrollar por parte del alumnado.

Prever los criterios metodológicos que utilizarán (secuencia de tareas, agrupamiento del alumnado, etc., ) en el desarrollo de las lecciones.

Establecer criterios para la evaluación formativa y sumativa de los procesos de enseñanza y aprendizaje realizados, que permitan informarse e informar a otros profesores, alumnado y familias.

\section{DESARROLLO}

Adecuar los contenidos a las posibilidades de aprendizaje del alumnado.

Utilizar los espacios, objetos, cadencia en las propuestas, etc., de tal manera que el alumnado se mantenga activo e implicado.

Trabajar con todos y con cada uno de los miembros del grupo.

Posibilitar al alumnado la evaluación de su propio trabajo, ¿ofrece criterios de éxito? ¿permite administrar los errores?, ¿ayuda a fijar los aprendizajes? etc.

ANÁLISIS

Establecer relaciones entre lo programado y lo ocurrido.

Incluir en sus argumentos y reflexiones las sugerencias y datos que le aportan los observadores externos.

Señalar principios (por ejemplo: el niño en el centro de las preocupaciones profesionales; Hacia una educación comprensiva; Hacia un tratamiento equilibrado y equitativo del alumnado; Hacia la igualdad de oportunidades en el alumnado, etc.) desde los que argumentar, reflexioen

Extraer datos relevantes para la información a las familias y el ejercicio de la tutoría.

Cuando lo presentamos en el Grupo de Trabajo comprobamos que, a pesar de que parcialmente era conocido, visto en su conjunto provocaba una serie de comentarios e inquietudes originales y, bajo mi punto de vista, muy valiosas. Veamos algunas de ellas:

- Señala lo mucho que me falta por aprender.

- Deja ver la enorme complejidad de nuestro trabajo.

- Me permite ver el trabajo que desarrollo en un marco general.

- Señala las tareas sobre las que debo canalizar mis esfuerzos.

Se trataba de un documento construido en los debates que se iban estableciendo en el Grupo de Trabajo y en las conversaciones con los estudiantes a la vuelta de su estancia en los centros escolares, pero al concre- 
tarlo del modo expuesto, se convertía en una referencia que permitía evaluar el desarrollo profesional y, más concretamente, el grado de formación, que tanto estudiantes como maestros, decían tener en relación con las competencias señaladas.

Animados por estas comprobaciones, convertimos e documento en una encuesta en la que se preguntaba, en una escala de 1 a 5 , por el nivel de formación que creían tener en cada una de las competencias señaladas, a la vez que se ofertaba un espacio en blanco para escribir sobre cómo creían que podría mejorar su formación en cada una de ellas.

Estas actividades se realizaron en el tercer trimestre del curso 2000-2001. Sus resultados nos permitieron orientar los proyectos, tanto de las asignaturas "Educación Física en Educación Primaria" y "Prácticas I", como el previsto para el Grupo de Trabajo en el nuevo curso 2001-2002.

Sabemos bien que toda formación es contextual y que lo surgido con nuestros estudiantes y con los maestros noveles y veteranos, especialistas y polivalentes, que forman el Grupo de Trabajo, es particular de estos colectivos, debido a ello, quizás sea el proceso seguido y la posibilidad que ofrece a otros estudiantes y maestros de bilidad que ofrece a otros estudiantes y maestros de ñaladas, lo que puede tener mayor interés para el lector.

Quisiera, no obstante, continuar un poco más apuntando algunas de las repercusiones que han tenido lugar en el Grupo de Trabajo.

El Grupo de Trabajo "Tratamiento Pedagógico de lo Corporal" se reúne desde hace algo más de una década los miércoles por la tarde en la Escuela Universitaria de Educación de Palencia. Desde hace dos años centra su trabajo en el desarrollo de una concreta Programación Genera Anual (Vaca Escribano, 2001). Dicha Programación General Anual hace que las reuniones del Grupo se organicen por quincenas. Un miércoles el trabajo consiste en inter cambiar sobre el tema señalado en la Programación General Anual y, el siguiente, en debatir sobre la exposición que algún miembro del grupo hace sobre lo ocurrido al respecto en su particular contexto educativo.

Pues bien, los resultados de la encuesta realizada sobre el documento de competencias nos invitan a:

Hacer hincapié en la coherencia interna de los elementos de la unidad didáctica a nivel de programación el porqué y para qué del tema elegido; objetivos y contenidos previstos; criterios metodológicos y actividades de enseñanza, aprendizaje y evaluación.

Por esta razón el maestro o la maestra encargada de la quincena expone el relato de lo ocurrido precedido de un proyecto en el que se justifica la unidad didáctica se precisan los aprendizajes a construir por parte de alumnado y las capacidades a desarrollar, y se relatan organizadas en una estructura de funcionamiento, las actividades y tareas a proponer.

Reflexionar sobre su posible concreción en los diferentes ciclos de la Educación Primaria.

Aunque el Proyecto y Desarrollo de la unidad didác tica expuesta se refieren a un nivel de enseñanza concreto, en el Análisis se hacen alusiones a su posible tratamiento en el resto de los ciclos de la Educación Primaria.
Identificar el singular significado del proceso de enseñanza y aprendizaje programado dentro de la Programación General Anual.

Los temas que componen la Programación General Anual suelen tener relaciones unos con otros. En el análisis de la unidad didáctica desarrollada se procura hacer hincapié en el singular significado del tema buscando que la Programación General Anual diversifique el contenido.

Pensar sobre sus repercusiones en el Proyecto Educativo de Centro.

Cualquiera que sea el tema tratado en la quincena reclama una reflexión sobre sus posibilidades de prolongación en otros momentos de la jornada escolar y extraescolar, lo que supone un material valioso para el intercambio entre el profesorado y la organización general del centro.

Por otra parte, el documento de competencias nos ayudará en el próximo mes de junio a analizar el trabajo desarrollado a lo largo del curso y concretar el nuevo proyecto, lo que supondrá, muy probablemente, concreciones y ajustes en el propio documento de competen cias.

Lamento que, una vez más, no ofrezcamos una información acabada, ni una pauta lista para ser aplicada, $n$ siquiera un conjunto de ideas generales tranquilizadoras de las que se desprende que todo va bien. El trabajo de formación de estudiantes y maestros en el ámbito corporal nos lleva a establecer procesos de este tipo, siempre inacabados, frágiles y complejos, que necesitan ser reconstruidos en cada contexto, e interpretados por cada maestro en su lugar de trabajo.

No obstante, el hecho de hacer públicas las competencias que se reclaman a los Maestros de Educación Física facilita sin duda el debate profesional y éste, su vez, ver con mayor claridad las posibilidades y dificultades de la formación del profesorado.

Todo está en cuestión, todo es susceptible de mejoray reformulación pero a su vez las competencias identificadas y las reflexiones que posibilitan favorecen que se pueda señalar con mayor precisión el contenido a impartir en Educación Física Escolar, ayudan a mejorar la calidad de los procesos de los procesos de enseñanza y aprendizaje y enriquecen el debate sobre la profesión de Maestro/a de Educación Física, y esto, por ahora nos es suficiente para seguir adelante.

\section{Bibliografía}

ELLIOTT, J. (1990): La investigación acción en educación. Editorial Morata. Madrid.

MINISTERIO DE EDUCACIÓN Y CIENCIA (1990): Ley Orgánica de Ordenación General del Sistema Educativo. Centro de Publicaciones. Madrid.

VACA ESCRIBANO, M. (2001): "El ámbito corporal en la Educación Primaria. Una propuesta curricular para el curso 2001-2002". Revista: AGORA PARA LA EDUCACIÓN FÍSICA Y EL DEPORTE. Valladolid. 2001 Páginas 56-71. 